\section{ORIGINAL RESEARCH}

R.P. Gamss

S.E. Slasky

J.A. Bello

T.S. Miller

S. Shinnar

\title{
Prevalence of Hippocampal Malrotation in a Population without Seizures
}

BACKGROUND AND PURPOSE: Hippocampal malrotation (HIMAL) is a failure of hippocampal inversion that occurs during normal fetal development and has been seen on MR imaging examinations of people with epilepsy, but it has not been studied in patients without epilepsy. We intended to evaluate the prevalence of HIMAL in MR imaging examinations of patients without seizures to better understand the significance of HIMAL in the population with seizure.

MATERIALS AND METHODS: A total of 497 MR imaging examinations with thin-section imaging through the temporal lobes of patients referred for conditions other than seizures were reviewed. The examinations were performed on 1.5T magnets. Sagittal T1-weighted and coronal T2-weighted images were used to evaluate each MR image for the distinctive features of HIMAL. As previously described in the literature, the criteria for HIMAL include unilateral involvement and incomplete rotation of a hippocampus that is normal in size and signal intensity but abnormally rounded in shape, with blurred inner structure. In addition, ipsilateral findings of an atypical collateral sulcus angle and atypical position and size of the fornix were noted. The corpus callosum is normal, and the temporal lobe remains normal in size, though the temporal horn may appear enlarged.

RESULTS: None of the patients' examinations fulfilled all of the HIMAL criteria. Six studies satisfied 2 or more criteria, which included an abnormally rounded hippocampus and a vertical collateral sulcus. These HIMAL findings were all seen on the left. Forniceal asymmetry was the most prevalent abnormality, with 289 patients manifesting a low position of 1 fornix.

CoNCLUSIONS: Hippocampal malrotation is a rare finding in patients without seizures. HIMAL is therefore likely to be a pathologic finding.

$\mathbf{T}$ he association between the hippocampus and epilepsy has been well studied. Temporal lobe epilepsy is the most common form of partial epilepsy, and hippocampal sclerosis is a common radiologic finding. ${ }^{1}$ It is also possible that developmental abnormalities of the hippocampus may predispose or otherwise contribute to epileptogenesis. ${ }^{2}$ In normal fetal development, the hippocampus inverts within the medial temporal lobe. In cases of hippocampal malrotation (HIMAL), hippocampal inversion fails to occur. A study published by Barsi et $\mathrm{al}^{3}$ found evidence of HIMAL in 32 of $527 \mathrm{MR}$ imaging examinations of patients with seizures, and suspected epilepsy. As part of the Consequences of Prolonged Febrile Seizures in Childhood (FEBSTAT) study ${ }^{4}$ we have reported the occurrence of HIMAL on MR imaging examinations performed in children with prolonged febrile seizures. ${ }^{5}$ These data suggest a possible role for HIMAL in the development of temporal lobe epilepsy. It is unclear whether HIMAL may predispose the hippocampus to insult, thus promoting the development of seizures and/or hippocampal sclerosis. To further evaluate the link between HIMAL and epilepsy, we sought to

\section{Received December 17, 2008; accepted after revision March 6, 2009}

From the Departments of Neurology, Pediatrics, and Epidemiology and Population Health (S.S.) and Department of Radiology (S.E.S., R.P.G., J.A.B., T.S.M.), Montefiore Medical Center, Bronx, NY.

Supported in part by grant NS 43209 from the National Institute of Neurological Disorders and Stroke (S.S., J.A.B.).

Previously presented as a poster at: American Society for Neuroradiology Annual Meeting and Neuroradiology Education and Research Foundation Symposium, May 31-June 5, 2008; New Orleans, La.

Please address correspondence to Todd Miller, Department of Radiology, Montefiore Medical Center, 111 E. 210th St, Bronx, NY 10467; e-mail: tmiller@montefiore.org

Indicates open access to non-subscribers at www.ajnr.org

DOI 10.3174/ajnr.A1657 establish the prevalence and significance of HIMAL in the population without seizures. We retrospectively reviewed 497 MR imaging studies that included thin-section coronal imaging through the temporal lobes, which were performed for various clinical indications other than seizures in patients from 2005 through 2007.

\section{Materials and Methods}

\section{Subjects}

Four hundred ninety-seven MR imaging examinations that included thin-section coronal imaging through the temporal lobes of patients referred for conditions other than seizures were reviewed. There were 154 male patients and 343 female patients with a ratio of 0.45 . Mean age of the patients was 51.7 years (age range, 15-101 years). However, most of the patients were between 25 and 75 years old.

The MR imaging examinations were performed for various clinical indications from 2005 through 2007. Half of the MR examinations were performed at an outpatient-imaging center that routinely does thin-section coronal imaging as part of a standard MR imaging protocol. Headache was the most common indication for the study patients' MR imaging studies (146/497). Among the patients whose indication was headache, $43 \%$ of them had an additional indication such as vision changes, dizziness, or vertigo. Some of the other common clinical indications were cerebrovascular accident or transient ischemic attack, memory loss, ataxia, vertigo, dizziness, dementia, weakness, and vision or hearing changes. We also reviewed the MR imaging examinations from patients with pituitary disorders who had imaging done at the hospital during these years.

\section{Imaging}

The examinations were performed on two $1.5 \mathrm{~T}$ magnets from the same vendor by use of 2 similar protocols. Half of the study popula- 


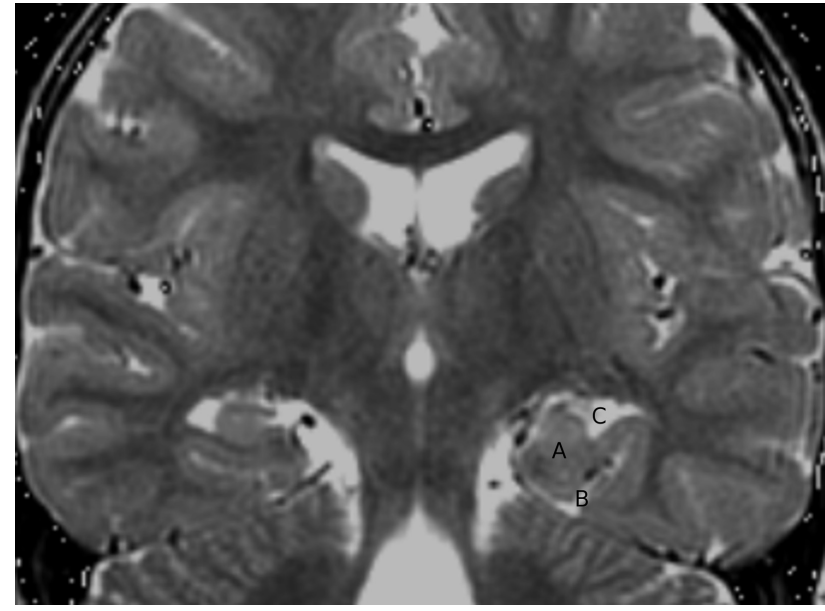

Fig 1. Coronal T2 MR image demonstrates an example of HIMAL. The right side is normal, but there is left-sided abnormal hippocampal formation. $A$, Rounded, medially displaced left side of the hippocampus. B, Vertical collateral sulcus. $C$, Enlarged-appearing temporal horn.

tion was recruited from a consecutive series from each magnet. Sagittal T1-weighted spin-echo (SE) imaging (TR, $480 \mathrm{~ms}$; TE, $13 \mathrm{~ms}$; flip angle, $69^{\circ}$; section thickness, $5 \mathrm{~mm}$; spacing, $6 \mathrm{~mm}$; matrix, $256 \times 256$ or TR, $450 \mathrm{~ms}$; TE, $14 \mathrm{~ms}$; flip angle, $69^{\circ}$; section thickness, $4 \mathrm{~mm}$; spacing, $5 \mathrm{~mm}$; matrix, $256 \times 256)$ and T2-weighted coronal SE imaging (TR, $5500 \mathrm{~ms}$; TE, $110 \mathrm{~ms}$; section thickness, $5 \mathrm{~mm}$; spacing, 6 mm; matrix, $256 \times 256$ or TR, $3000 \mathrm{~ms}$; TE, $80 \mathrm{~ms}$; section thickness, $3 \mathrm{~mm}$; spacing, $3.3 \mathrm{~mm}$; matrix, $256 \times 256$ ) were used to evaluate each subject for the presence of distinctive features of HIMAL. The coronal images were perpendicular to the long axis of the hippocampus.

Each study was independently reviewed by 2 board certified radiologists, and 1 medical student who was trained to recognize the distinctive features of HIMAL. Any potential HIMAL case was then reviewed by an adjudicating senior CAQ (Certification of Added Qualification) certified neuroradiologist and a board certified neurologist with experience in evaluating patients with seizures. Retrospective chart review or consultation with the patient's primary medical doctor was done for any potential HIMAL case.

As previously described by Barsi et $\mathrm{al}^{3}{ }^{3}$ the criteria for HIMAL include unilateral involvement and incomplete rotation of a hippocampus that is normal in size and signal intensity, but abnormally rounded in shape so that the dimensions in either direction are equal, with blurred inner structure. In addition, ipsilateral findings of an atypical collateral sulcus angle and atypical position and size of the fornix were noted. The normal collateral sulcus angle is flat at the level of the body and tail of the hippocampus. In cases of HIMAL, the collateral sulcus angle is more vertical. The normal position of the fornix is symmetrical. In cases of HIMAL, the fornix can be unilaterally downwardly displaced. The corpus callosum is normal, and the temporal lobe remains normal in size, though the temporal horn may appear enlarged (Fig 1). ${ }^{3}$

A $\chi^{2}$ was used to determine whether there was a significant difference between the prevalence of HIMAL in these patients referred for conditions other than seizures vs patients with seizures, as reported by Barsi et $\mathrm{al}^{3}$. Because of the small numbers in some cells, the Fisher exact test was used.

\section{Results}

None of the study patients' MR imaging examinations fulfilled all of the criteria for HIMAL. Fornix asymmetry was the most prevalent abnormality, with 58\% (289/497) of study patients manifesting a low position of 1 fornix. Of these, 77\% (223/ 289 ) had a low left fornix, and $23 \%(66 / 289)$ had a low right fornix. Five of the study MR images demonstrated 2 features of HIMAL without a low fornix, and 1 study demonstrated a single feature of HIMAL without a low fornix. Among the other criteria for HIMAL, an abnormally round hippocampus and/or vertical collateral sulcus were seen in 7 of the MR imaging studies. Retrospective chart review or consultation with the patients' primary care physicians was performed in these 6 patients with partial HIMAL features to verify that they do not have any history of seizures.

Statistical analysis with use of the Fisher exact test comparing our results (no HIMAL case in 497 patients referred for conditions other than seizures) with those of Barsi et $\mathrm{al}^{3}$ (32 HIMAL cases in 527 patients with seizures) was highly significant ( $P$ value of $4.19 \times 10^{-10}$ with a $95 \%$ confidence interval). Of the $6 \mathrm{MR}$ imaging studies with 2 or more HIMAL criteria, 1 demonstrated a low fornix and 5 demonstrated a normally positioned fornix. Of the 209 subjects with a normal fornix, 6 (2.9\%) demonstrated 1 or more HIMAL criteria; of the 289 with a low fornix, 1 subject $(0.35 \%)$ demonstrated additional HIMAL criteria.

Our cohort of patients had a female predominance that was not fully explained because there was no selection on the basis of sex.

\section{Discussion}

This retrospective review demonstrates that hippocampal malrotation is nearly nonexistent in patients referred for MR imaging for conditions other than seizures. However, there were limitations to our study. The prevalence of HIMAL reported by Barsi et $\mathrm{al}^{3}$ served as the reference point for our calculations because, to our knowledge, there is limited additional literature indicating the prevalence of HIMAL. We used a similar protocol to that of the study by Barsi et $\mathrm{al}^{3}{ }^{3}$ and we used their list of the common imaging characteristics of HIMAL in our evaluation of HIMAL. In analyzing our data, we compared our observations with those of their study. Potential bias existed because we may have implemented the HIMAL criteria differently.

The study by Barsi et $\mathrm{al}^{3}$ does not provide information for patients who did not fulfill all of the HIMAL criteria. Therefore, the percentage of patients without HIMAL but with a low fornix is unknown. These data might further clarify the exact significance of the fornix position as a HIMAL criterion. Because a low fornix was prevalent in $58 \%$ of our patients without seizures and without HIMAL, we hypothesize that a low fornix might be a normal variant. A low fornix is not associated with any known neurologic sign or symptom. Additional research may elucidate whether a low-positioned fornix is pertinent to the HIMAL criteria vs a normal variant.

Because $2.9 \%$ of our subjects with a normally positioned fornix had 1 or more HIMAL criteria and $0.35 \%$ with an associated low fornix had 1 or more HIMAL criteria, it is plausible that a low fornix may be incidental.

The FEBSTAT study, ${ }^{4}$ a prospective multicenter study of febrile status epilepticus funded by the National Institutes of Health, examined the presence of hippocampal abnormalities 
on MR images of children with prolonged febrile seizures. In a preliminary analysis of the early cases, HIMAL was found in 9 of 61 febrile status cases and in 3 of 56 control cases with more brief febrile seizures. ${ }^{5}$ All of these cases of HIMAL occurred on the left side. The previous findings, which will be confirmed in the larger cohort of 200 that FEBSTAT will ultimately recruit, suggest that HIMAL is a pathologic finding because it was found more commonly in the children with prolonged febrile seizures. Follow-up imaging of these children should help determine whether HIMAL predisposes them to temporal lobe epilepsy and mesial temporal sclerosis. ${ }^{5}$

Our study did not address the effect of age on the rounded appearance of the hippocampus. Our purpose was not to conduct a longitudinal study of the normal hippocampus but, rather, to address the prevalence of HIMAL. A separate study would be required to assess how the hippocampus changes with age.

A similar study design sought to determine the presence of mesial temporal sclerosis on MR imaging examinations of patients without seizures. The study concluded that mesial temporal sclerosis is uncommon in the population without seizures and should prompt investigation of an underlying seizure disorder. ${ }^{6}$ In a likewise fashion, our study has shown that HIMAL is also uncommon in the population without seizures and should prompt investigation of an underlying disorder.

\section{Conclusions}

HIMAL is a rare finding in patients referred for MR imaging for conditions other than seizures. An imaging finding of HIMAL should therefore raise suspicion of an underlying potential for or the presence of a seizure disorder. Our study failed to demonstrate an association between HIMAL criteria and a low-lying fornix.

\section{References}

1. Meiners L, Gils A, Jansen G, et al. Temporal lobe epilepsy: the various MR appearances of histologically proven mesial temporal sclerosis. AJNR Am J Neuroradiol 1994;15:1547-55

2. Lehericy S, Dormont D, Semah F, et al. Developmental abnormalities of the medial temporal lobe in patients with temporal lobe epilepsy. AJNR Am J Neuroradiol 1995;16:617-26

3. Barsi P, Kenez J, Solymosi D, et al. Hippocampal malrotation with normal corpus callosum: a new entity? Neuroradiology 2000;42:339-45

4. Shinnar S, Hesdorffer DC, Nordli DR Jr, et al. Phenomenology of prolonged febrile seizures: results of the FEBSTAT study. Neurology 2008;71:170-76

5. Lewis DV, Chan S, Bello JA, et al. HIMAL is a malformation that predisposes to prolonged febrile seizures: data from the FEBSTAT Study. Epilepsia 2006; 47(Suppl 4):16

6. Moore KR, Swallow CE, Tsuruda JS. Incidental detection of hippocampal sclerosis on MR images: is it significant? AJNR Am J Neuroradiol 1999;20:1609-12 